Bull. Mater. Sci., Vol. 20, No. 6, September 1997, pp. 739-744. (C Printed in India.

\title{
Lattice dynamics and molecular dynamics simulation of complex materials
}

\author{
S L CHAPLOT \\ Solid State Physics Division, Bhabha Atomic Research Centre, Mumbai 400 085, India
}

\begin{abstract}
In this article we briefly review the lattice dynamics and molecular dynamics simulation techniques, as used for complex ionic and molecular solids, and demonstrate a number of applications through examples of our work. These computational studies, along with experiments, have provided microscopic insight into the structure and dynamics, phase transitions and thermodynamical properties of a variety of materials including fullerene, high temperature superconducting oxides and geological minerals as a function of pressure and temperature. The computational techniques also allow the study of the structures and dynamics associated with disorder, defects, surfaces, interfaces etc.
\end{abstract}

Keywords. Molecular dynamics simulation; lattice dynamics; phase transitions; ionic solids; fullerene; high-temperature superconductors

\section{Introduction}

The Gibb's free energy of a crystalline phase has terms corresponding to the potential energy, the pressure volume $P V$, the vibrational energy and the vibrational entropy (Born and Huang 1954). Thus, at $P=0$ and $T=0$, the minimization of the potential energy largely determines the stability, although the zero-point vibrational energy may also sometimes contribute significantly. The relative stability of phases at a finite temperature can be obtained if the phonon spectra in the different phases are also evaluated (Chaplot 1987). Various thermodynamical properties, including the equation of state and specific heat, can be calculated in this way.

The quasi-harmonic lattice dynamics technique can be used up to a temperature beyond which the anharmonicity of the vibrations becomes too large. At very high temperatures the dynamics may not be described in terms of phonons, and phenomena such as diffusion and molecular reorientation become important. In such cases, explicit computer simulation of the structure and the dynamics using the technique of the molecular dynamics (Rahman 1964) is found to be very useful in providing a microscopic insight into the behaviour of the materials. Molecular dynamics also allows conveniently to study the structure and dynamics associated with defects, disorder, melting etc.

The phonon spectra, diffusion kinetics in terms of the geometry and the time scales, etc can in general be derived from experiments (Chaplot 1994) such as neutron scattering experiments. However, a theoretical modelling of the phenomena is necessary in studies on complex structures at both the planning and analysis stages of the experiments. Thus a suitable combination of the experiment and theory is required to provide a fundamental understanding of the material behaviour. 


\section{Techniques}

It is well known that the technique of lattice dynamics (Born and Huang 1954; Venkataraman et al 1975; Pawley 1986) essentially involves setting up a dynamical matrix of force constants and its diagonalization to get the phonon frequencies and the polarization vectors. Molecular dynamics simulation (Rahman 1964; Rao and Chaplot 1979; Chaplot 1986; Allen and Tildesley 1987), on the other hand, involves explicitly integrating the equations of motion to get the atomic trajectories of motion.

The key ingredient in simulations is a suitable interatomic potential function for the classical simulations (Parrinello and Rahman 1980) and a first principle total energy calculation for the first principles-simulation (Car and Parrinello 1985). The latter approach is a bit too difficult at present for complex materials, which have a number of atomic species interacting with a combination of a variety of interactions such as the long-ranged ionic potentials, van der Waals and anisotropic covalent potentials. The state-of-the-art for complex materials is to use appropriate (semi)empirical potential functions, though the limitation on their usage and transferability to different environments have to be kept in mind. In many cases, semi-empirical potentials have been successfully used to derive qualitative or semi-quantitative useful information under new bonding environments in new phases (Chaplot and Sikka 1993a, b), at surface (Pawley and Chaplot 1980) and in small clusters.

We have derived empirical potentials and used them for a variety of complex materials, e.g. molecular ionic solids, high-temperature superconducting oxides, geological minerals and organic solids, including fullerene.

At Trombay we have developed the necessary softwares DISPR for lattice dynamics (Chaplot 1978) and MOLDY for molecular dynamics simulations (Chaplot 1986, unpublished), which may be applicable to systems of arbitrary size and symmetry. In particular, these softwares are also applicable to systems containing molecular ions for which the long-ranged Coulomb interaction is handled via the Ewald technique. Recent MD simulations have been performed on periodic cells of up to 30000 particles using the BARC-parallel computer ANUPAM.

\section{Results and discussion}

\subsection{Complex ionic solids}

In our early lattice dynamics work on the ionic solids based on a rigid molecular ion model for $\mathrm{KNO}_{3}$ (Rao et al 1978), $\left(\mathrm{NH}_{4}\right)_{2} \mathrm{CuCl}_{4} \cdot 2 \mathrm{H}_{2} \mathrm{O}$ (Chaplot and Sahni 1979), $\mathrm{LiKSO}_{4}, \mathrm{LiNbO}_{3}, \mathrm{KNbO}_{3}$ (Chaplot and Rao 1983), $\mathrm{Sb}_{2} \mathrm{~S}_{3}$ and SbSI (Rao and Chaplot 1985), the empirical potentials were determined largely by the static and dynamic equilibrium conditions and found to predict most phonon frequencies to a discrepancy level of about $10 \%$, which was much better than might be expected. Our work on $\mathrm{KNO}_{3}$ was perhaps the first example (Chaplot et al 1977; Rao et al 1978) of an empirical potential for an ionic molecular solid, derived and used for prediction of all the rigid body vibrations. The potential model involved about two parameters for each atom type, corresponding, respectively, to the long-range 
Coulomb and the short-range repulsive interaction. We found that the parameters could be further tuned, or additional interactions such as the hydrogen bond included, to improve the agreement with experiment.

The potential for $\mathrm{LiKSO}_{4}$ was also successfully used in molecular dynamics simulation to understand the nature of dynamics of the sulphate reorientations (Chaplot and Rao 1987), and the pressure-induced crystalline and amorphous structural transitions (Chaplot and Sikka 1993a). Lattice dynamics calculation at high pressure also allowed to identify the soft phonon modes, occurring prior to the structural transition (Chaplot and Sikka 1993a, b).

We also refer to a recent MD simulation of solid palladium deuteride (Rao and Chaplot 1996) in which attention was focussed on short-lived large-energy fluctuations of atoms in the solid. It is pointed out that such fluctuations occur at a significant rate of order $1 \mathrm{THz}$ and could have consequences for unusual events such as 'cold fusion'.

\subsection{High-temperature superconductors}

High-temperature superconducting oxides are important materials in which we could make early predictions (Chaplot 1988), prior to the experiments, of the phonon dispersion relation; the predictions were excellent for the low energy phonons in $\mathrm{YBa}_{2} \mathrm{Cu}_{3} \mathrm{O}_{7}$ and were quite fair in the high energy region with an average discrepancy level of about $10 \%$. Our model predictions for $\mathrm{Tl}_{2} \mathrm{CaBa}_{2} \mathrm{Cu}_{2} \mathrm{O}_{8}$ (Chaplot et al 1991; Chaplot 1992), which included the effect of oxygen positional disorder through the use of a supercell, were successfully tested against our neutron inelastic scattering measurement of the phonon density of states at the Dhruva reactor at Trombay.

The empirical potential was used in MD simulations (Chaplot 1989, 1990) to understand the oxygen disordering transition and diffusion (figure 1) at high temperatures in $\mathrm{YBa}_{2} \mathrm{Cu}_{3} \mathrm{O}_{7}$. It is observed that the oxygen diffusion occurs through
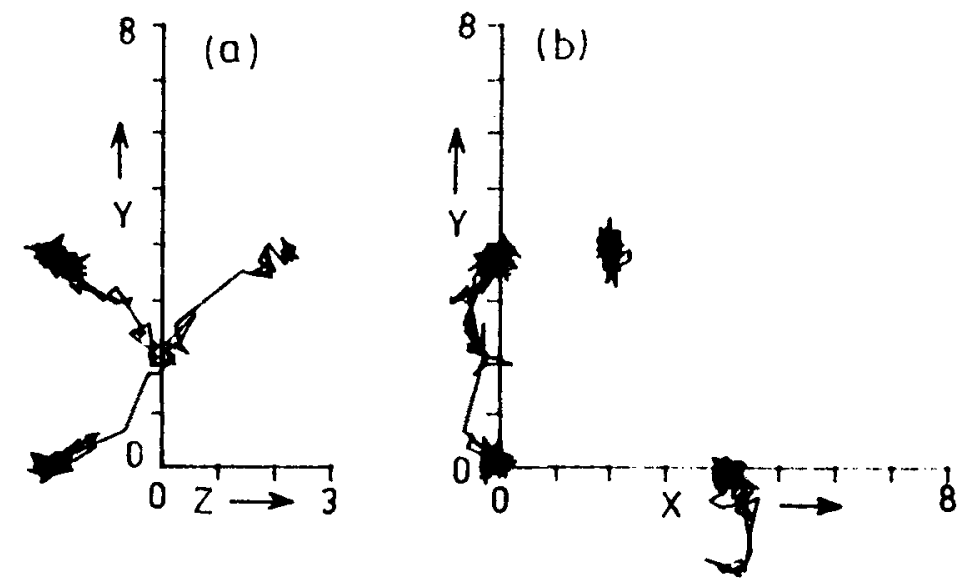

Figure 1. Molecular dynamics simutation of oxygen jump-diffusion in the high-femperature superconductor $\mathrm{YBa}_{2} \mathrm{Cu}_{3} \mathrm{O}_{7}$ it $1100 \mathrm{~K}$. The $\mathrm{YZ}$ and $\mathrm{XY}$ projections of the coordinates of certain atoms for a duration of $10 \mathrm{ps}$ are shown (Chaplot 1989). The angstrom units are used. 
hopping among the available octahedral sites. Due to its simplicity and the level of accuracy, the empirical potential for $\mathrm{YBa}_{2} \mathrm{Cu}_{3} \mathrm{O}_{7}$ was also found useful by others for a variety of applications such as the calculation of the eiectron-phonon coupling (Zeyher 1990) and phonon contribution in neutron scattering (Fong et al 1995).

More recently, we have improved the model (Chaplot et al 1995b) by including the shell model interactions, and by fitting to a large data base of phonon dispersion relation in a number of related systems, with average discrepancy level of 3-4\% only. This work helped to focus attention on phonon anomalies, which could not be explained through traditional lattice dynamics alone and may be attributed to electron-phonon coupling etc.

\subsection{Geological minerals}

Magnesium silicates $\left(\mathrm{Mg}_{2} \mathrm{SiO}_{4}\right.$ and $\mathrm{MgSiO}_{3}$ ) form the predominant constituents of the Earth's mantle and occur in a variety of structural phases at different depths down to $2700 \mathrm{~km}$. Seismic discontinuities may occur due to compositional or structural phase changes. With an aim to understand the phase transitions and the thermodynamic properties we have investigated a number of phases.

Our early systematic work on forsterite, $\mathrm{Mg}_{2} \mathrm{SiO}_{4}$ ( $\mathrm{Rao}$ et al 1987, 1988), involving neutron inelastic scattering and lattice dynamics, led to a simple model framework which allowed to calculate the thermodynamic properties (Choudhury et al 1989). Later similar work was also carried out on two of the enstatite phases (Ghose et al 1993; Choudhury et al 1997). Now we have calculated the phase diagram for all the four enstatite phases using the free energy approach and a common interatomic potential (Choudhury et al 1996). The calculations are in qualitative agreement with experiments, and show that the ortho- to proto-enstatite transition as $P=0$ and the clino- $P 22_{1} / c$ to clino- $C 2 / c$ enstatite transitions at $P=8 \mathrm{GPa}$ occur at high temperature driven by the vibrational entropy, while the ortho to clino transition is pressure driven.

Molecular dynamics simulation in silicate minerais (Chaplot et al 1996) have been carried out to understand the microscopic mechanisms and the kinetics of transitions. The simulations have also led to predictions of new phases and phase transitions, e.g. the orthoenstatite characterized by $\mathrm{MgO}_{6}$ octahedra and $\mathrm{SiO}_{4}$ tetrahedra is first found to transform to a phase containing $\mathrm{SiO}_{5}$ units and then at higher pressures to another phase with $\mathrm{MgO}_{8}$ and $\mathrm{SiO}_{6}$ units as in the orthorhombic perovskite.

It is perhaps for the first time that transformations from the low-pressure phases existing in the Earth's upper mantle to the high-pressure phases existing in the lower mantle have been simulated. The observed seismic discontinuities in the Earth's mantle are believed to be either due to phase transitions or changes in the chemical composition. The simulations need to be carried out more extensively on larger cells, and can be expected to produce a very useful understanding of the seismic discontinuities and the complex phase diagram of the silicates.

We have also simulated the perovskite $\mathrm{MgSiO}_{3}$ at high pressures and temperatures to investigate the diffusion of oxygens and melting. It is observed in the simulations that the lattice defects in the crystal significantly enhance the diffusion process while the $\mathrm{Mg} / \mathrm{Fe}$ substitutional disorder has a negligible effect (Chaplot et al 1996). 


\subsection{Fullerene}

An important application of the lattice dynamics and molecular dynamics techniques has been in the study of the rigid molecular structure and dynamics of fullerene (Chaplot and Pintschovius 1995; Pintschovius et al 1995). This work also required important developments of the intermolecular potential function. The known van der Waals potential predicts the correct lattice constant, bulk modulus and the cohesive energy. However, this potential could not explain the true ground state among many competing states of nearly equal energies, the unexpectedly large librational frequencies and the high orientational disordering temperature. A number of models involving electrostatic interaction of bond charges etc were developed which explained some of the above features. We developed a split-bond-charge model which produced a very good description of the phonon dispersion relation of the lattice modes as measured by neutron inelastic scattering (Pintschovius and Chaplot 1995)

We also used this model in molecular dynamics simulation of the orientationally disordered phase of fullerene occurring at high temperatures. The neutron-quasielastic-diffuse scattering patterns, as generated by the simulated dynamical structure, produced an excellent agreement with experiments. It is the partial short-range order (extending up to several neighbours) in the disordered phase (or, in other words, the orientational correlations of the neighbouring fullerene molecules), which give rise to a very rich pattern showing anisotropy and inhomogeneity of the diffuse scattering in the wave-vector space. The time-scale (of several picoseconds) at which the correlations are created and destroyed results in the energy scale of the neutron quasielastic scattering (of a few meV). In fact, the energy $(E)$ scale is correlated to the wave-vector $(Q)$ via the dynamical structure factor $S(Q, E)$, which is the Fourier transform of the space-time correlation function $G(r, t)$. The MD simulation was used in deriving the dynamical structure in the disordered phases and indicated the presence of the strong double bond-pentagon correlations. The simulation also reproduced the average single particle number density as derived from the usual X-ray and neutron Bragg diffraction experiments. We note that in the case when the diffuse scattering arises from static disorder it may be analysed using a reverse Monte-Carlo approach (Chaplot et al 1995a). The molecular dynamics is preferable in the case of dynamical behaviour.

In summary, we have given brief account of some of the computational techniques with examples for the study of a variety of dynamical behaviour in complex materials.

\section{References}

Allen M P and Tildesley D J 1987 Computer simulation of liquids (Oxford: Clarendon)

Born M and Huang K 1954 Dynamical theory of crystal lattices (Oxford: Clarendon)

Car R and Parrinello M 1985 Phys. Rev. Lett. $55247 \mathrm{l}$

Chaplot S L 1978 Report B.A.R.C. 972

Chaplot S L 1986 Curr. Sci. 55949

Chaplot S L 1987 Phys. Rev. B36 8471

Chaplot S L 1988 Phys. Rev. B37 7435

Chaplot S L 1989 Phase Transitions 1949 
Chaplot S L 1990 Phys. Rev. B42 2149

Chaplot S L 1992 Phys. Rev. B45 4885

Chaplot S L 1994 Indian J. Pure \& Appl. Plys. 32560

Chaplot S L and Sahni V C 1979 Phys. Status Solidi (b) 96575

Chaplot S L and Rao K R 1983 J. Phys. C: Solid State Phys. 163045

Chaplot S L and Rao K R 1987 Phys. Rev. B35 9771

Chaplot S L and Pintschovius L 1995 Fullerene Sci. \& Technol. 3707

Chaplot S L and Sikka S K 1993a Phys. Rev. B47 5710

Chaplot S L and Sikka S K 1993 b Plys. Rev. Lett. 712674

Chaplot S L, Rao K R, Venkatesh A H and Vijayaraghavan P R 1977 Nucl. Phys. Solid State Phys. (India) C20 277

Chaplot S L, Dasannacharya B A, Mukhopadhyay R, Rao K R, Vijayaraghavan P R, lyer R M, Phatak G M and Yakhmi J V 1991 Physica B174 378

Chaplot S L, Pintschovius L, Haluska M and Kuzmany H 1995a Phys. Rev. B51 17028

Chaplot S L, Reichardt W, Pintschovius L and Pyka N 1995b Pliys. Rev. B52 7230

Chaplot S L, Choudhury N and Rao K R 1996 International conference on condensed matter under high pressure. Mumbai (eds) S K Sikka, S C Gupta and B K Godwal (New Delhi: National Institute of Science Communication) (in press)

Choudhury N, Chaplot S L and Rao K R 1989 Phys. Chem. Miner. 16599

Choudhury N, Chaplot S L and Rao K R 1996 International ('onference on condensed matter under high pressure, Mumbai (eds) S K Sikka, S C Gupta and B K Godwal (Now Delhi: National Institute of Science Communication) (in press)

Choudhury N, Ghose S, Chowdhury C P, Loong C K and Chaplot S L 1997 Phys. Rev. B

Fong H F, Keimer B, Anderson P W, Reznik D, Dogan F and Aksay I A 1995 Phys. Rev. Lett. 75 316

Ghose S, Choudhury N, Chaplot S L, Chowdhury C P and Sharma S K 1993 Phys. Chem. Miner. 20 469

Parrinello M and Rahman A 1980 Phys. Rev. Lett. 451196

Pawley G S 1986 in Methods of experimental physics (eds) K Skold and D Price (New York: Academic Press) A23 p. 44 I

Pawley G S and Chaplot S L 1980 Phys. Status Solidi (b)99 517

Pintschovius L and Chaplot S L 1995 Z. Phys. 98527

Pintschovius L, Chaplot S L, Roth G and Heger G 1995 Phys. Rev. Lett. 752843

Rahman A 1964 Phys. Rev. A136 105

Rao K R and Chaplot S L 1979 in Current trends in lattice dynunics (ed.) K R Rao (Bombay: Indian Physics Association) p. 589

Rao K R and Chaplot S L 1985 Phys. Status Solidi (b)129 471

Rao K R and Chaplot S L 1996 Fusion Technol. 30355

Rao K R, Chaplot S L, lyengar P K, Venkatesh A H and Vijayaraghavan P R 1978 Pramana-J. Phys. 11251

Rao K R, Chaplot S L, Choudhury N, Ghose S and Price D L 1987 Science 23664

Rao K R, Chaplot S L, Choudhury N, Ghose S, Hastings J M, Corliss L M and Price D L 1988 Phys. Chem. Miner. 1683

Venkataraman G, Feldkamp L and Sahni V C 1975 Dynamics of perfect crystals (Boston: MIT Press)

Zeyher R 1990 Z. Phys. B80 187 